\title{
Trust Evaluation Model for Internet of Things using Mobile Ad hoc Networks
}

\author{
*1Stephen A. Mogaji, ${ }^{2}$ Olaniyi A. Ayeni and ${ }^{3}$ Oluwaseun G. Fadare \\ ${ }^{1}$ Department of Computer Science, Federal Technical College, Ikare, Nigeria \\ ${ }^{2}$ Department of Cyber Security, Federal University of Technology, Akure, Nigeria \\ ${ }^{3}$ Department of Computer Science, Joseph Ayo Babalola University, Ikeji Arakeji, Nigeria \\ sdepomogaji@gmail.com | oaayeni@futa.edu.ng | fadareadelodun90@gmail.com
}

ORIGINAL RESEARCH ARTICLE

Received: 13-AUG-2021; Reviewed: 01-OCT-2021; Accepted: 01-NOV-2021

http://dx.doi.org/10.46792/fuoyejet.v6i4.685

\begin{abstract}
Internet of Things (IoT) is a theory that embraces various objects and methods of communication to exchange information. Today, IoT is more a descriptive term of a vision that everything should be linked to the internet. As many as the advantages of loT, its uniqueness poses a new problem in establishing a trustworthy environment amid some of the items due to the lack of appropriate implementation machineries. However, some common network security measures are not adequate to preserve the integrity of information and services exchanged over the internet. As a result, they remain vulnerable to threats ranging from cyber-physical layer and social layer data management risks. IoT in smart environments interrelates with mobile ad hoc network (MANET), becoming even more attractive and economically successful. This research proposes two different metrics approaches namely social trust metrics and Quality of Service to evaluate the trustworthiness of loT Applications using MANET nodes and designing trust metrics that are computed using multiple properties of trust and quality of service. A model would be designed and simulated in NS-2. The findings of the investigation into the evaluation of trustworthiness and network performance are presented. The proposed system model has $99.31 \%$ accuracy when there were $10 \%$ malicious nodes in the network. When the number of misbehaving nodes was increased to $50 \%$, the accuracy level dropped to 98.75 percent, a difference of only $0.56 \%$. The results show that the security and trustworthiness of the proposed system is an improvement over the existing models like Collaborative Reputation (CORE) trust model and Ant Based Evidence Distribution (trust) model. The proposed model can be used to address security challenges in a network environment.
\end{abstract}

Keywords- Confidence, Honesty, IoT, MANET, QoS, Trustworthiness

\section{INTRODUCTION}

$\mathrm{T}$ he Internet of Things (IoT) and cloud computing typify a future internet concept. With developing communication technologies, objects (such as real or virtual things) can recognize and interact with each other at any time, allowing enhanced services to be provided over global infrastructure in several aspects of daily life (Jain et al., 2012). Mobile Ad-hoc Network (MANET) is similar to wireless sensor networks in that each node acts as a router or a network end system (WSNs). The interplay of MANET and IoT opens up new avenues for service provision in smart settings while also posing challenges in terms of networking.

In this work, a model of trustworthiness for the mobile IoT system using MANET is proposed. The model which is mainly focused on social trust metric and Energy level consumption which is the Quality of Service (QoS) in a simulated environment using NS2. MANET network nodes were selected as a router head and a reactive routing protocol was used (Mogaji 2018). The Internet of Things (IoT) is a network of "things" with sensors that will be able to connect and share data over network links, and each thing will be uniquely identified by its integrated computing system The Internet of Things enables "things" to be intuited and controlled from afar (Waleed et al., 2019). In order to improve privacy, trust is a crucial component of access restriction Gowrishankar et al., 2020).

${ }^{*}$ Corresponding Author

Section B- ELECTRICAL/ COMPUTER ENGINEERING \& RELATED SCIENCES Can be cited as:

Mogaji S.A., Ayeni O.A., and Fadare O.G. (2021): Trust Evaluation Model for Internet of Things using Mobile Ad hoc Networks, FUOYE Journal of Engineering and Technology (FUOYEJET), 6(4), 353-357. http://dx.doi.org/10.46792/fuoyejet.v6i4.685

\section{LITERATURE REVIEW}

In years past, different trust management models have been proposed to enhance security in MANETs to enable nodes to evaluate their neighbours directly or through recommendations from other nodes in the network.

i.CORE (COllaborative REputation) by Michiardi and Molva (2002), Cooperation of Nodes-Fairness in Dynamic Ad-hoc NeTworks (CONFIDANT) by Buchegger and Le Boudec (2004).

ii.Ant Based Evidence Distribution (ABED) model by Jiang \& Baras (2006)

iii.Singh, et al. (2019) proposed an IoT and MANET trustbased model to estimate the trust value by combining direct and indirect trust.

iv.Merlin \& Ravi (2019) developed a trust-based, energyconscious routing mechanism. This method effectively handles only the black hole detection multi detection routes.

These are system level-based trust models which support cooperation in ad hoc networks by detecting and isolating malicious nodes. These models being system level-based trust model omit some important evaluation metrics such as quality of service and social properties in evaluating nodes' trustworthiness. This is considered a research gap in which the focus of such models is directed toward a single parameter only in computing trustworthiness.

\section{Materials AND Methods}

A trust-based management framework has been made to enable nodes to establish a trustworthy route and reliable data delivery in IoT-MANET. In MANETs, optimal trustworthiness evaluation demands multidimensional properties which show human behaviour in addition to QoS. In this paper, social and QoS properties are used to assess node trustworthiness. The metrics used to calculate the trustworthiness of nodes are listed below. 


\subsection{PositiVe INTERACTION}

Positive interaction (or feedback) is a social factor in MANETS, defined as the total number of forwarding packets delivered successfully by a node in the network. This value is represented as $\rho$. Accumulated positive interaction is calculated as

$$
\alpha=\rho+1 \text {. }
$$

\subsection{NEgATIVE INTERACTIONS}

Negative interaction, in contrast to positive feedback, is a significant social factor measured by the number of packets dropped by a network node. This value is represented as $n$. Accumulated negative interaction, $\beta$ is calculated as

$$
\beta=n+1
$$

\subsection{HONESTY}

Honesty is a social property and a friendship-based trust model metric. It refers to the degree of honesty of the evaluating node $j$ about the evaluated node $i$. It is a measure of successful or failed interactions. Negative and positive behaviours of nodes are indicators of the honesty of nodes in detecting irregular behaviour. The value of Honesty, $H_{i j}$ is calculated by using the number of successful interactions $\alpha_{i j}$ between node $i$ and $j$ over the maximum number of successful and failed interactions, $\alpha_{i j}+\beta_{i j}$.

$$
H_{i j}=\frac{\alpha_{i j}}{\alpha_{i j}+\beta_{i j}}
$$

The initial value of $H_{i j}$ is 0.5 at time $t=0$, which means that node $i$ is a stranger to node $j$ and no previous interaction has been observed. $H_{i j}$ develops over time also, and its value is between 0 and 1 . Positive interactions increase the value of honesty while negative interactions can lead to a decrease in its value.

\subsection{ConfIDENCE MetRIC}

Another friendship-based trust metric is confidence, which is an important social property that indicates how strong a link exists between two interacting nodes. It evaluates the strength of relationships between interacting nodes by measuring how frequently nodes interact with one another. As a result, the evaluating node's ability to judge the node under evaluation's trustworthiness improves (Shabut, 2015).

Confidence $C_{i j}$ is expressed as the variance value of all past experiences between two interacting nodes. Its value is measured by using the beta standard deviation, $\sigma$

$$
\begin{aligned}
C_{i j} & =1-\sqrt{12 \sigma_{i j}} \\
\sigma_{i j} & =\frac{\alpha_{i j} \times \beta_{i j}}{\left(\alpha_{i j}+\beta_{i j}\right)^{2}\left(\alpha_{i j}+\beta_{i j}+1\right)}
\end{aligned}
$$

The beta standard deviation equation has been rewritten to normalize its values over a range of values $[0,1]$ using the constant $1-\sqrt{12 \sigma_{i j}} . \quad \alpha_{i j}$ and $\beta_{i j}$ represents the positive and negative interaction observed by node $i$ and $j$. When time $t=0$ node $i$ is not able to judge the honesty of node $j$ even if its honesty value is more than a trust threshold, $\alpha_{i j}$ and $\beta_{i j}$ is 1 and $C_{i j}=0$. The updated value of $\alpha_{i j}$ and $\beta_{i j}$ will be calculated as $\alpha_{i j}=\rho+1$ and $\beta_{i j}=$ $n+1$, where $\rho$ and $n$ represent the positive and negative collected observations respectively, and $\rho$ and $n \geq 0$.

\subsection{ENERGY LEVEL}

In trust systems, energy is a critical Quality of Service (QoS) factor. All nodes are energy constrained, and each node's lifetime is determined by its energy consumption. In the proposed model, the Energy Level, $V_{i j}$ factor indicates the remaining energy level of the node after each trust update interval $t$ performed by the evaluating node $i$ about the evaluated node $j$. The energy factor is calculated as

$$
V_{i j}=\frac{E_{i j}-F_{i j}}{G_{i j}}
$$

Where,

$E_{i j}$ is the previous current energy of node $j$,

$F_{i j}$ is the level of energy consumed by node $j$ in performing interactions, and

$G_{i j}$ is the initial level of energy of node $j$ to start with.

The nodes in the network start with the equal energy level. The only types of communications that are measured using energy consumption are receiving and transmitting packets. The quantity of the energy level is defined in the range $[0,1]$. It starts at 1 , indicating that nodes have a fully charged battery, and gradually decreases over time as nodes engage in more communications. Nodes continue to be effective in performing interactions in as much as the energy level is not reduced to a particular threshold.

\subsection{Trust Model Objective Function for IoT- MANETS}

The cost function is evaluated using multidimensional social trust and QoS metrics describes above. Positive interaction, negative interaction, honesty, and confidence level are social trust metrics, while the energy factor is an important QoS metric. As a result, the trustworthiness is assessed by combining all metrics, as shown in Figure 1. The confidence factor, $C_{i j}$, measures the level of experience between interacting nodes. The honesty factor, $H_{i j}$, measures selfishness or maliciousness of nodes. The energy factor, $V_{i j}$, measure if a node is capable of performing an intended task or not. The threshold for all multidimensional metrics introduced in this work is 0.5 . The proposed IoT- MANET trust model architecture is shown in Figure 1.

$$
T_{i j}=\frac{H_{i j}+C_{i j}+V_{i j}}{3}
$$

\subsection{EVAluating TRUSt IN IOT-Manets USING PROPOSED SYSTEM MODEL}

The greater the Trust value, the better the performance of the proposed system. The trustworthiness $T_{i j}$ is therefore evaluated by combining all metrics, setting the threshold for all multidimensional metrics to 0.5 and according to equation 7 , any node whose trust value or cost value is evaluated to be less than the set threshold which is 0.5 is considered to be malicious or misbehaving node. 


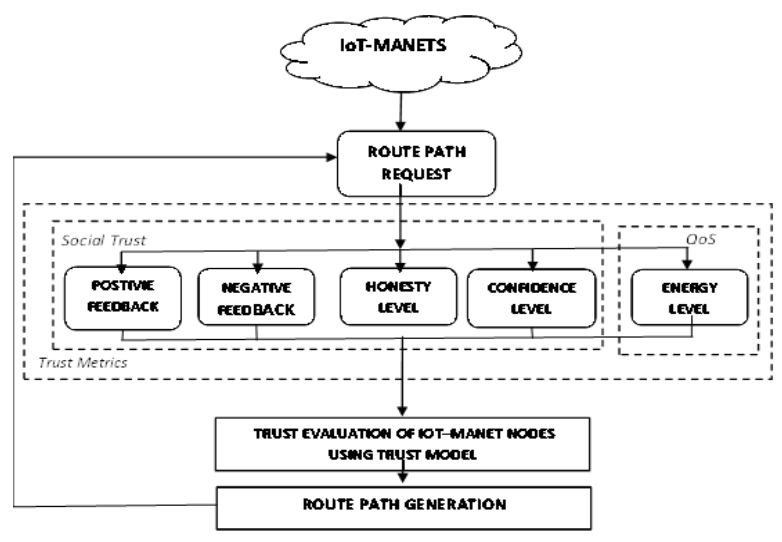

Fig. 1: The proposed loT- MANET trust model architecture. (Adapted from Mogaji et al, 2018)

\subsection{THE EXPERIMENTAL SETUP}

A network with 50 randomly placed nodes was simulated. Several nodes were randomly selected to be misbehaving by dropping packets at different rates. Table 1 shows the parameters used in configuring the network for the experiment. The misbehaving nodes dropped the packets at random while the good nodes dropped the packets due to the environment of the MANET in the simulation. In the network, bad-behaving accounted for up to $50 \%$ of the packets dropped.

As a result, if a node's trust threshold value falls below 0.5 due to excessive packet drop and other malicious activities, the node is considered bad. Results from the experiment used to evaluate the proposed model are based on summarized multiple runs, and negligible variation.

\subsection{THE NETWORK SimULATOR}

There are many tools available for simulations of network topologies. But the proposed algorithm is simulated using the Network Simulator 2 (NS-2) tool.

Table 1. Simulated Network Configurations

\begin{tabular}{cc}
\hline Parameter & Values \\
\hline Number of Nodes & 50 \\
Speed & $10 \mathrm{~m} / \mathrm{s}$ \\
Routing Protocol & AODV \\
MAC & 802.11 \\
Source-destination & 15 \\
Pairs & \\
Transmitting Capacity & $2 \mathrm{Kbps}$ \\
Packet Size & $512 \mathrm{~B}$ \\
Simulation & $500 \mathrm{~s}$ \\
Trust Threshold & 0.5 \\
\hline
\end{tabular}

\section{RESULTS AND DISCUSSION}

The results and analysis of the computation of the trust model's components, namely the social Trust metrics and QoS trust metric values, are presented in this section. The values are also provided, as well as their relationship to the overall trustworthiness value. The simulation results over a number of iterations are shown in Table 2.
Table 2. Overall trustworthiness of the proposed system

\begin{tabular}{cc}
\hline Number of Iteration & $\begin{array}{c}\text { Overall } \\
\text { Trustworthiness }\end{array}$ \\
\hline 30 & 0.90413 \\
40 & 0.90623 \\
50 & 0.92867 \\
60 & 0.93197 \\
70 & 0.93400 \\
80 & 0.95127 \\
90 & 0.95810 \\
100 & 0.95787 \\
\hline
\end{tabular}

The graphical representation of the values of the social trust and QoS trust components used to produce the composite trust metric of the proposed system in relation to the number of interactions are presented in Figure 2

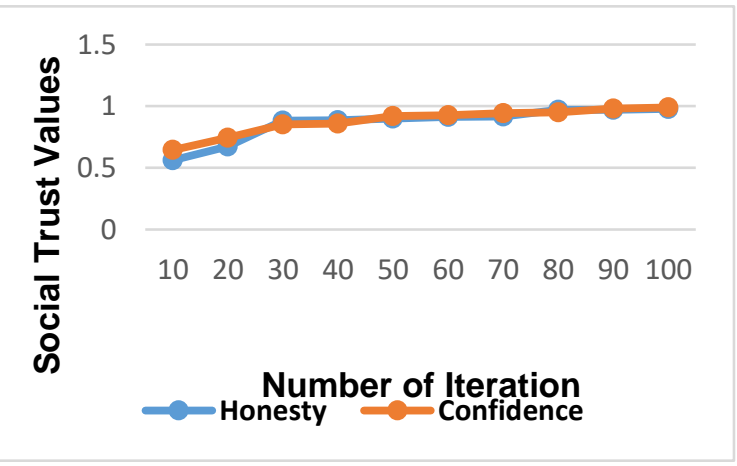

Fig. 2: Social trust value metric of the proposed system with number of iteration.

Figure 2 demonstrates the value of social components: honesty and confidence. Figure 3 shows, on the other hand, that as the evaluated node becomes more involved in network interactions, its estimated QoS in the form of energy value decreases. Figure 4 below shows the overall trustworthiness value of the proposed system.

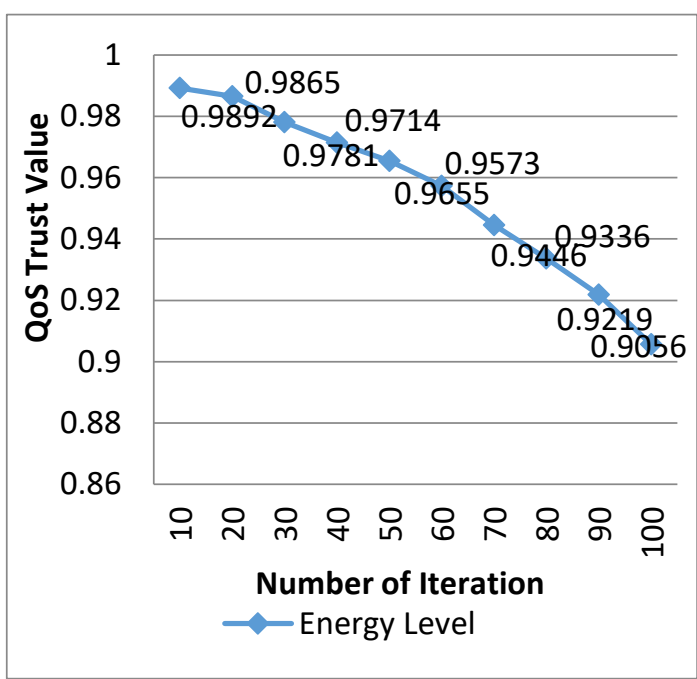

Fig. 3: QoS trust value metric of the proposed system with number of iterations 


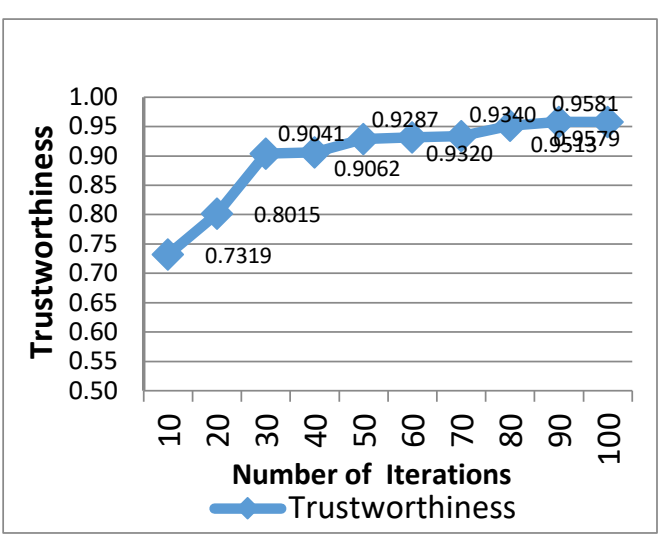

Fig. 4: The value of the composite trustworthiness combining all trust metrics of the proposed system with number of iterations

\section{Node Evaluation}

The figure 5 presents the evaluation of the proposed model in accurately evaluating trusted node in the network environment and choosing good nodes for communication path generation. It shows that as the number of misbehaving nodes increase in a network environment, the accuracy slightly reduces but at a very minute rate.

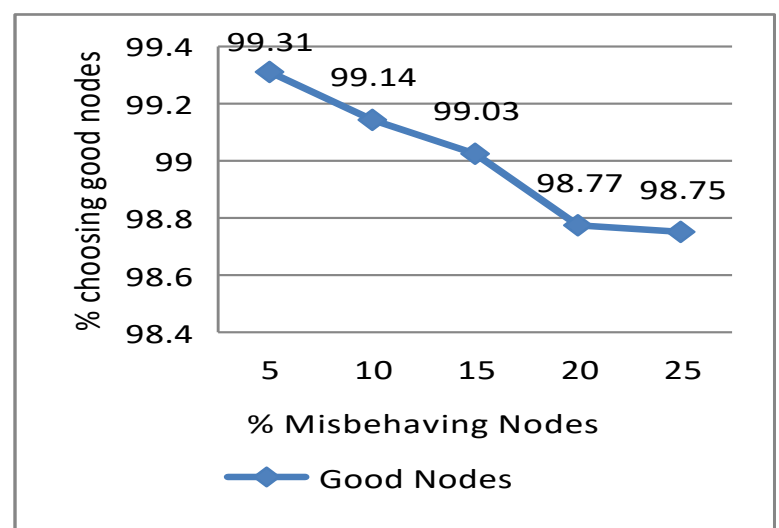

Fig. 5: Effective node evaluation in the presence of misbehaving nodes for the proposed system

When 5 malicious nodes representing $10 \%$ of the entire nodes existed in the network, the proposed system model has an accuracy of $99.31 \%$. Increasing the misbehaving nodes to 25 nodes representing $50 \%$ of the entire node, the accuracy level dropped slightly to $98.75 \%$, representing a mere $0.56 \%$ difference.

\section{Comparative Analysis of the Proposed MODEL WITH OTHER TWO EXISTING TRUST MODELS}

This section shows performance comparison between the proposed system and two of other existing trust models as shown in Table 3 below.

Collaborative Reputation (CORE) trust model is systembased trust model that distinguish the selfish node and misbehaving node. ABED is Ant Based Evidence Distribution model. It is protocol-based trust model. It is a model in which agents discovered the best way to calculating evidence of trust. These two models adopted single parameters in computing trustworthiness and this is considered a research gap upon which the proposed model of this research work is built. Using multi- dimensional trust metrics in computing trustworthiness consequently made it perform better than the two models as shown in the table below.

Table 3. Comparative analysis of effective node evaluation in the presence of misbehaving nodes for proposed model and two other existing trust models.

\begin{tabular}{cccc}
\hline $\begin{array}{c}\text { Number of } \\
\text { Malicious nodes }\end{array}$ & \multicolumn{3}{c}{ \% choosing good nodes } \\
& $\begin{array}{c}\text { CORE } \\
\text { model }\end{array}$ & $\begin{array}{c}\text { ABED } \\
\text { model }\end{array}$ & $\begin{array}{c}\text { Proposed } \\
\text { model }\end{array}$ \\
\hline $\mathbf{1 0}$ & 76.79 & 88.57 & 99.31 \\
$\mathbf{2 0}$ & 72.25 & 86.53 & 99.14 \\
$\mathbf{3 0}$ & 70.04 & 83.87 & 99.02 \\
$\mathbf{4 0}$ & 68.84 & 82.06 & 98.77 \\
$\mathbf{5 0}$ & 63.95 & 79.98 & 98.75 \\
\hline
\end{tabular}

\section{ConcLusion}

The proposed system optimization model for trust management system to determine the trustworthiness of participating nodes in mobile ad-hoc networks was tested against benchmark functions to prove its correctness and quick convergence edge. The model was then incorporated into a simulated IoT-MANET environment using specially configured parameters and from the network analysis.

This model is recommended for distributed network environments that are vulnerable to network attacks, where security and trust are important, as well as lowenergy devices, such as those found in IoT smart environments. This model also optimises energy level of nodes, which promises to increase the life-span of communication devices used in emergency operations for disaster relief.

\section{FUTURE WORK}

In this research work, a set of social and QoS properties of trust were used to model the behaviour of nodes in MANETs. These models can be extended by using more social and QoS properties to detect any malicious or bad behaving like newly joined nodes or changing identities.

\section{REFERENCES}

Buchegger, S., and Le Boudec, J.Y. (2004). A Robust Reputation System for P2P and Mobile Ad hoc Networks P2P and Mobile Ad-hoc Networks, Wireless Personal Communications, vol. 37, pp. 23-26.

Gowrishankar J, Senthil Kumar P, Narmadha T, Yuvaraj N. (2020). A Trust Based Protocol for Manets in IOT Environment. International Journal of Advanced Science and Technology, 29(7), 2770-2775

Jain S, Baras J. (2012) Preventing wormhole attacks using physical layer authentication". In Proceedings of the 2012 IEEE Wireless Communications and Networking Conference (WCNC), Shanghai, China, pp. 2712-2717.

Jiang Theodorakopoulos and J.S. Baras, (2006) “On Trust Models and Trust Evaluation Metrics for Ad-hoc Networks," IEEE Journal on selected Areas in Communications, Vol24, No. 2, Feb 2006, pp. 318-328.

Kamel M.B.M, Alameri I, Onaizah A.N. (2017). STAODV: A Secure and Trust based Approach to Mitigate Blackhole Attack on AODV based MANET", IEEE 2nd Advanced Information Technology, Electronic and Automation Control Conference, pp. 1278-1282.

Merlin, R.T., \& Ravi, R. (2019). Novel Trust Based Energy Aware Routing Mechanism for Mitigation of Black Hole Attacks in 
MANET. Wireless Personal Communications,104(4), 1599-1636.

Michiardi, P., and Molva, R. (2002). CORE: A COllaborative REputation mechanism to enforce node cooperation in Mobile Ad Hoc Networks, Communications and Multimedia Security Conference, pp. 107-121.

Mogaji, S.A (2018). Security and Trust Model using Swarm Intelligence system for Mobile Adhoc Networks. Ph.D thesis submitted to the Department of Computer Science, Federal University of Technology Akure, Nigeria.

Mogaji S. A., Alese B. K., Adetunmbi, A. O. and Olabode, O. (2018). Development of Trust metrics using Swarm Intelligence Techniques for Mobile Adhoc Networks. International Journal of Scientific \& Engineering Research Volume 9, Issue 12, ISSN 22295518. pp.1324-1334.

Singh, U., Shukla, M., Jain, A. K., Patsariya, M., Itare, R., \& Yadav, S. (2019). Trust Based Model for Mobile Ad-Hoc Network in Internet of Things. In International Conference on Inventive Computation Technologies (pp. 827-839). Springer, Cham.

Shabut A.R.M (2015). Trust Computational Models for Mobile Ad Hoc Networks, a Ph.D. thesis submitted to School of Computing Faculty of Engineering and Informatics University of Bradford.

Waleed Alnumay, Uttam Ghosh, Pushpita Chatterjee (2019). A TrustBased Predictive Model for Mobile Ad Hoc Network in Internet of Things Sensors 2019, 19, 1467; doi:10.3390/s19061467. pp. 1-14. 